\title{
Effect of Lutzomyia whitmani (Diptera: Psychodidae) Salivary Gland Lysates on Leishmania (Viannia) braziliensis Infection in BALB/c Mice
}

\author{
Haroldo Sérgio da S Bezerra ${ }^{+}$, Maria Jania Teixeira
}

Núcleo de Medicina Tropical Prof. Joaquim Eduardo de Alencar, Faculdade de Medicina, Universidade Federal do Ceará, Rua Alexandre Baraúna 949, 60430-160 Fortaleza, CE, Brasil

Previous reports showed that Lutzomyia longipalpis saliva exacerbate Leishmania braziliensis infection in mice. The sand fly Lu. whitmani is one of the vectors of $\mathrm{L}$. (Viannia) braziliensis ( $\mathrm{LVb}), a$ causative agent of cutaneous leishmaniasis in the State of Ceará, Brazil. To determine whether saliva of $\mathrm{Lu}$. whitmani could increase the infectivity of $\mathrm{LVb}$ in mice, we inoculated groups of BALB/c mice with $\mathrm{LVb}$ promastigotes in the presence or absence of the salivary glands lysate from $\mathrm{Lu}$. whitmani. We found that coinjection with $\mathrm{Lu}$. whitmani saliva increased size but not longevity of cutaneous $\mathrm{LVb}$ lesions in BALB/C mice, since the formed lesions gradually resolved. The mechanism(s) by which Lu. whitmani saliva might exacerbate $\mathrm{LVb}$ infection in $B A L B / c$ mice is speculated.

Key words: Leishmania braziliensis - saliva - Lutzomyia whitmani - BALB/c mice

Leishmaniases are parasitic diseases caused by protozoan parasites of the genus Leishmania that produce either tegumentary (cutaneous, mucocutaneous, diffuse) or visceral clinical presentations in man and are responsible for significant morbidity and mortality in many areas throughout the world (Pearson et al. 1999). The mammalian host becomes infected with Leishmania when the sand fly vector, Phlebotomus species in the Old World and Lutzomyia species in the New World, probes the skin of parasitized host for a blood meal and injects the parasite admixed with its saliva (Titus et al. 1994).

Previous studies have shown that salivary gland lysates of Lu. longipalpis and P. papatasi markedly enhance the infectivity of $L$. major in mice (Titus \& Ribeiro 1988, Theodos et al. 1991). This exacerbating effect on the course of cutaneous leishmaniasis applies not only to infection with $L$. major, causative agent of Old World cutaneous leishmaniasis, but also to infection with other parasites causing cutaneous leishmaniasis in the New

\footnotetext{
This work was supported by Organização Panamericana da Saúde and Fundação Nacional de Saúde

${ }^{+}$Corresponding author. Fax: +55-85-288.8316. E-mail: pato@ufc.br

Received 15 May 2000

Accepted 5 October 2000
}

World, such as L. mexicana (Theodos et al. 1991) and L. braziliensis (Samuelson et al. 1991, Lima $\&$ Titus 1996). Additionally, it has been shown that the enhancement of infectivity is not due to a direct effect of the saliva on the parasite but rather to an effect on the host. Sand fly saliva has several pharmacological activities, including vasodilation, inhibition of platelet aggregation, and inhibition of coagulation (Ribeiro 1987). Besides, sand fly saliva may also have immunomodulatory effects related to the pathogenesis of leishmanial infections (Hall \& Titus 1995, Qureshi et al. 1996, Mbow et al. 1998).

We report here the effect of salivary gland lysate of Lu. whitmani on the course of $L$. (V.) braziliensis infection in BALB/c mice. The rationale for extending these observations to a model using $L$. (V.) braziliensis plus salivary gland lysate from $L u$. whitmani is that this sand fly is the most important natural vector for $L$. (V.) braziliensis in Ceará, Brazil (Queiroz et al. 1994). Moreover, $L$. $(V$.) braziliensis is transmitted in nature neither by $\mathrm{Lu}$. longipalpis nor P. papatasi, the sand fly species targeted on saliva studies up to now.

The $L$. (V.) braziliensis strain (MCAN-BR-9219914) used in this study was maintained in Schneider's insect medium (Sigma, St. Louis, MO) supplemented with $10 \%$ heat-inactivated fetal calf serum (Sigma), 2\% sterile normal human urine, $100 \mathrm{U} / \mathrm{ml}$ penicillin (Sigma), $100 \mu \mathrm{g} / \mathrm{ml}$ streptomycin (Sigma) and $2 \mathrm{mM}$ L-glutamine (Gibco 
BRL, Grand Island, NY) at $26^{\circ} \mathrm{C}$. Parasites were used at no later than the fourth passage. Salivary gland lysates were obtained from sugar-fed 3-5day-old adult laboratory-reared, female $L u$. whitmani from a closed laboratory colony maintained at Núcleo de Medicina Tropical/UFC and used as described previously (Titus \& Ribeiro 1988).

When L. (V.) braziliensis promastigotes were inoculated with salivary gland lysate, the lesion appeared around seven days post-infection. They peaked in size at 28 days of evolution and persisted for up to 42 days, then regressed in a slower way than the lesions of the controls without saliva (Figure). Also, in the animals coinjected with $L$. (V.) braziliensis plus $\mathrm{Lu}$. whitmani saliva, the lesions developed always larger than those of the controls did, which it was statistically significant $(p<0.05)$ at virtually all time point examined (Figure).

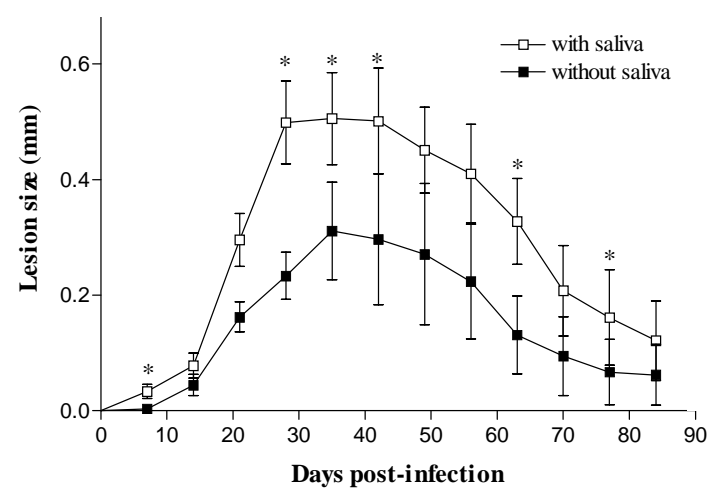

Enhancement of Leishmania (Viannia) braziliensis (LVb) infection in BALB/c mice by coinjection of parasites with Lutzomyia whitmani salivary glands lysates. Groups of nine $\mathrm{BALB} / \mathrm{c}$ mice were injected subcutaneously in the right hind footpad with $10^{7}$ stationary phase $\mathrm{LVb}$ promastigotes in 20 $\mathrm{ml}$ sterile saline with or without the lysate of 0.5 of one salivary gland per foot. Lesion sizes were measured weekly with a dial gauge caliper and expressed as the difference in thicknesses $(\mathrm{mm})$ of the infected and contralateral uninfected footpads; values represent the mean $\pm \mathrm{SE} ; * p<0.05$.

In the BALB/c model of infection, $L$. braziliensis induces only transient and non-severe cutaneous lesion. Nonetheless, when L. braziliensis parasites are injected into BALB/c mice with sand fly saliva, they cause progressive skin lesions and infection is significantly enhanced as measured by lesion size, parasitic burden, and outcome of infection (Samuelson et al. 1991, Lima \& Titus 1996, Donnelly et al. 1998). In this work we found that coinjection of $L$. (V.) braziliensis plus Lu. whitmani salivary gland lysate also increases the size of cutaneous lesions in BALB/c mice. However, while in the works using coinjection of Lu. longipalpis saliva plus $L$. (V.) braziliesies the cutaneous lesions persisted for the lifetime of the mice (Samuelson et al. 1991, Lima \& Titus 1996), we showed that coinjecting saliva of $L u$. whitmani plus $L$. (V.) braziliensis it formed spontaneously resolving cutaneous lesions. The mechanism(s) by which $\mathrm{Lu}$. whitmani saliva exacerbates $L$. (V.) braziliensis infection in BALB/c mice may only be speculated, since most of the salivary factor(s) responsible for the enhancement of infectivity of Leishmania have not yet been identified. In the saliva of $L u$. longipalpis one key molecule is the peptide maxadilan, which besides being a potent vasodilator may also have immunomodulatory properties (Lerner et al. 1991, Qureshi et al. 1996, Soares et al. 1998). P. papatasi lacks maxadilan, but its saliva contains large amounts of adenosine and 5'AMP (Ribeiro et al. 1999). It has been demonstrated that adenosine is probably the factor in $P$. papatasi that interferes with the ability of activated macrophages to kill parasites (Katz et al. 2000).

So far as we are aware, the present report is the first to show that salivary gland lysate of $L u$. whitmani enhance the infectivity of $L$. (V.) braziliensis in mice. Our data support other studies that have demonstrated that the vectors' saliva somehow modulates the long-term pathology of the disease. We hope with this work to contribute for a better understanding of the role of $L u$. whitmani saliva in the natural transmission of $L$. (V.) braziliensis cutaneous leishmaniasis in this region of the world.

\section{ACKNOWLEDGMENTS}

To Antônio Wilson Vasconcelos and Eddie WP Santana for revising the manuscript.

\section{REFERENCES}

Donnelly KB, Lima HC, Titus RG 1998. Histologic characterization of experimental cutaneous leishmaniasis in mice infected with Leishmania braziliensis in the presence or absence of sand fly vector salivary gland lysate. J Parasitol 84: 97-103.

Hall LR, Titus RG 1995. Sand fly vector saliva selectively modulates macrophage functions that inhibit killing of Leishmania major and nitric oxide production. J Immunol 155: 3501-3506.

Katz O, Waitumbi JN, Zer R, Warburg A 2000. Adenosine, AMP, and protein phosphatase activity in sandfly saliva. Am J Trop Med Hyg 62: 145-150.

Lerner EA, Ribeiro JMC, Nelson RJ, Lerner MR 1991. Isolation of maxadilan, a potent vasodilatory peptide from the salivary glands of the sand fly Lutzomyia longipalpis. J Biol Chem 266: 1123411236.

Lima HC, Titus RG 1996. Effects of sand fly vector saliva on development of cutaneous lesions and the immune response to Leishmania braziliensis in $\mathrm{BALB} / \mathrm{c}$ mice. Infect Immun 64: 5442-5445. 
Mbow ML, Bleyenberg JA, Hall LR, Titus RG 1998. Phlebotomus papatasi sand fly salivary gland lysate down-regulates a Th1, but up-regulates a Th2 response in mice infected with Leishmania major. $J$ Immunol 161: 5571-5577.

Pearson RD, Jeronimo SMB, Queiroz AS 1999. Leishmaniasis. In RL Guerrant, DH Walker, PF Weller (eds), Tropical Infections Diseases. Principles, Pathogen \& Practice, Churchill Livingstone, Philadelphia, p. 797-813.

Queiroz RG, Alencar IAB, Vasconcelos AW, Pessoa FAC, Sousa RN, David JR 1994. Cutaneous leishmaniasis in Ceará state in Northeastern Brazil: incrimination of Lutzomyia whitmani (Diptera: Psychodidae) as a vector of Leishmania braziliensis in Baturité municipality. Am J Trop Med Hyg 50: 693698.

Qureshi A, Asahina A, Ohnuma M, Tajima M, Granstein $\mathrm{R}$, Lerner E 1996. Immunomodulatory properties of maxadilan, the vasodilator peptide from sand fly salivary gland extracts. Am J Trop Med Hyg 54: 665671.

Ribeiro JMC 1987. Role of saliva in blood-feeding by arthropods. Annu Rev Entomol 32: 463-478.

Ribeiro JMC, Katz O, Pannell LK, Waitumbi J, Warburg
A 1999. Salivary glands of the sand fly Phlebotomus papatasi contain pharmacologically active amounts of adenosine and 5'-AMP. J Exp Biol 202: 1551-1559.

Samuelson J, Lerner E, Tesh R, Titus R 1991. A mouse model of Leishmania braziliensis braziliensis infection produced by coinjection with sand fly saliva. $J$ Exp Med 173: 49-54.

Soares MB, Titus RG, Shoemaker CB, David JR, Bozza M 1998. The vasoactive peptide maxadilan from sand fly saliva inhibits TNF-alpha and induces IL-6 by mouse macrophages through interaction with the pituitary adenylate cyclase-activating polypeptide (PACAP) receptor. J Immunol 160: 1811-1816.

Theodos CM, Ribeiro JM, Titus RG 1991. Analysis of enhancing effect of sand fly saliva on Leishmania infection in mice. Infect Immun 59: 1592-1598.

Titus RG, Ribeiro JM 1988. Salivary gland lysates from the sand fly Lutzomyia longipalpis enhance Leishmania infectivity. Science 239:1306-1308.

Titus RG, Theodos CM, Shankar A, Hall LR 1994. Interactions between Leishmania major and macrophages. In B Zwilling, T Eisenstein (eds), Macrophage Pathogen Interactions, Marcel Dekker, New York, p. 437-459. 
